\title{
A Stable Multi Agent based Optimization Approach for Load Shedding Control in Power Systems
}

\author{
Nila P Divakaran \\ PG scholar in Power Systems Engineering \\ Vivekanandha College of Engineering for Women \\ Elayampalayam, Tamilnadu
}

\author{
A Dyaneswaran \\ Assistant Professor in Electrical and Electronics \\ Department \\ Vivekanandha College of Engineering for Women \\ Elayampalayam, Tamilnadu
}

\begin{abstract}
This paper presents new approaches to update our existing load shedding scheme, planned for the coming years. If generation in power system is insufficient to power all loads, efficient load shedding operations may need to be deployed to maintain the supply demand balance. This paper proposes a distributed multi agent based Particle Swarm Optimization, which can make the efficient load shedding decision based on the global information. This method integrates the multi agent system and PSO algorithm. In order to obtain optimal solution quickly, each agent competes and corporate with its neighbors, and it can also learn by using its knowledge. According to this algorithm, total net active power and operating status of loads can be discovered accurately even with faults.
\end{abstract}

\section{Keywords}

Multi Agent System, Particle swarm optimization, Load shedding, Voltage Stability

\section{INTRODUCTION}

The Objectives of Power Systems designs are high power, electrical utility applications, industrial and commercial applications. Power Management is the aspect of managing the electrical loads such as available power loads are not overloaded The important objective of power systems design for high power, electrical utility applications, industrial and commercial applications. Power management is the aspect of (1) managing the electrical loads such as available power loads are not overloaded. (2) Power is allocated to the different loads such that the loads are receiving a proper allocation of power. Power management gives the good idea about Electrical Demand and Supply.

Power Management Systems (PMS) are essential for safe, efficient and reliable operation of industrial power systems. The functionality of the PMS suite includes load-shedding, power sharing, network synchronization and power restoration. PMS solutions protect and optimize the stability of industrial power systems in disturbance situations by ensuring power sharing between generators when the industrial power system is islanded from the grid. These solutions also ensure that the generators meet the required power demand when the network is connected to the grid [1]. The load shedding functionality ensures power availability to critical process loads by dropping less critical loads.

The load shedding problem has a significant influence on secure and economic operation of power systems [2]. Suppose any faults, sudden load change, and insufficient generation can create power mismatch between generation and loads. Load shedding is the process of tripping certain amount of load with lower priority to maintain the stability of the remaining portion of system.

Different methods have been proposed for load shedding. Multi Agent System is one of the most popular distributed control solutions [12]. This control scheme requires the collection and transmission of global information. Advantages of MAS include the ability to survive single point failures and decentralized data processing, which leads to efficient task distribution. It causing faster operation and decision making process

The Multi Agent system and Particle Swarm Optimization are integrated to form the proposed MAPSO method for solving load shedding problem. In MAPSO not only a solution to the optimization problem but also a particle to PSO. According to this method, two agents will communicate with each other only if their corresponding buses are connected. Through information exchanges, each agent can discover necessary global information for load shedding decision making. PSO is used to optimize the information exchange. Then the load shedding activities of all agents can be coordinated. Find out the optimum value of the load to be shed.

Multi Agent Based PSO is used for controlling the load shedding problem. Faults, sudden load change and insufficient generation can create major problems between generation and loads [9]. If generation is insufficient, efficient load shedding operations may need to be maintaining the supply demand balance. It focuses interactions between a utility company and its customers/users

This method proposed to determine the most appropriate loads to be shed during under frequency and under voltage condition. Total net active power can be calculating and operating status of loads can be estimating accurately even with faults. It based on the discovered information; coordinated Load shedding decision can be made.

\section{PROBLEM FORMULATION}

Load shedding problem can be formulated as an optimization problem with the following objective function and constraints [3].

\subsection{Objective function}

The Objective Function of the load shedding problem is to minimize the sum of curtailed load during generation outage conditions. It can be expressed mathematically as:

$$
\min f(t)=\sum_{i=1}^{N b u s} \alpha i . \Delta P d i \wedge 2+\beta i . \Delta Q d i \wedge 2 \ldots \ldots \ldots \ldots \ldots \ldots \ldots \ldots \ldots \ldots \ldots \ldots . .1
$$


Where $\alpha_{\mathrm{i}}$ and $\beta_{\mathrm{i}}$ are the weight factors for curtailed active and reactive power load of the ith bus and Nbus is the number of buses in the transmission system. $\Delta \mathrm{P}_{\mathrm{di}}$ and $\Delta \mathrm{Q}_{\mathrm{di}}$ is the curtailed active and reactive power load of the ith transmission system.

\subsection{Constraints}

The constraints can be listed as follows:

- Power flow balance equations:

$$
\begin{aligned}
& \mathrm{P} g i-\mathrm{P} d i 0-\Delta \mathrm{P} d i-V i \sum_{j=1}^{\mathrm{N}} V j Y i j \cos (\delta i-\delta j-\theta i j)=0 \ldots \ldots \ldots \ldots \ldots . . .2 \\
& Q g i-Q d i 0-\Delta Q d i-V i \sum_{j=1}^{\mathrm{N}} V j Y i j \cos (\delta i-\delta j-\theta i j)=0 \ldots \ldots \ldots \ldots . . .3
\end{aligned}
$$

$\mathrm{P}_{\mathrm{gi}}$ and $\mathrm{Q}_{\mathrm{gi}}$ are active and reactive power generations at the ith bus. $\mathrm{P}_{\mathrm{di} 0}$ and $\mathrm{Q}_{\mathrm{di} 0}$ is initial active and reactive power load of the ith bus. V's and $\delta$ 's, are system bus voltages magnitudes and phase angles. $Y_{\mathrm{ij}}$ and $\theta_{\mathrm{ij}}$ are bus admittance matrix elements.

- Maintaining the original load power factor:

To maintain the original load power factor $\Delta \mathrm{Q}_{\mathrm{di}}$ is selected as

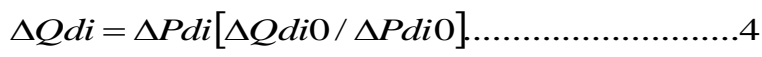

- Generators active and reactive power limits:

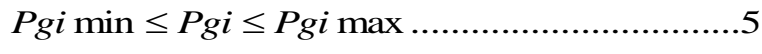

Qgi $\min \leq Q g i \leq Q g i \max \ldots \ldots \ldots \ldots \ldots \ldots \ldots \ldots \ldots \ldots$

- Voltage range limits:

$V i \min \leq V i \leq V i \max$ 7

- Line loading limits:

$|\delta i-\delta j| \leq \varepsilon i j$

Where $\delta_{\mathrm{i}}$ and $\delta_{\mathrm{j}}$ are the voltage angles at bus $\mathrm{i}$ and bus $\mathrm{j}$, and $\varepsilon_{\mathrm{ij}}$ is the maximum voltage phase angle difference between buses $i$ and $\mathrm{j}$.

\section{MULTI AGENT BASED PARTCLE SWARM OPTIMIZATION}

\subsection{Particle Swarm Optimization}

Particle Swarm Optimization (PSO) simulates the behaviors of bird flocking. Suppose the following scenario: a group of birds are randomly searching food in an area. There is only one piece of food in the area being searched. All the birds do not know where the food is. But they know how far the food is in each iteration. The effective one is to follow the bird, which is nearest to the food. PSO learned from the scenario and used it to solve the optimization problems.

In PSO, each single solution is a "bird" in the search space. We call it "particle". All of particles have fitness values, which are evaluated by the fitness function to be optimized, and have velocities, which direct the flying of the particles. The particles fly through the problem space by following the current optimum particles.
PSO is initialized with a group of random particles and then searches for optima by updating generations. In every iteration, each particle is updated by following two "best" values. The first one is the best solution (fitness) it has achieved so far. (The fitness value is also stored.) This value is called "pbest". Another "best" value that is tracked by the particle swarm optimizer is the best value, obtained so far by any particle in the population. This best value is a global best and called " $\mathrm{g}$ best". When a particle takes part of the population as its topological neighbors, the best value is a local best and is called p-best [11]. After finding the two best values, the particle updates its velocity and positions with following equation.

$$
\begin{aligned}
& \operatorname{Vi}(u+1)=W * V i(u)+C 1 * \operatorname{rand}() * \text { pbesti }-P i(u))+ \\
& C 2 * \operatorname{rand}() *(\text { gbesti }-\mathrm{Pi}(u)) \ldots \ldots \ldots \ldots \ldots \ldots \ldots \ldots \ldots \ldots \ldots \ldots \ldots . . . .9 \\
& P i(u+1)=P i(u)+V i(u+1) .
\end{aligned}
$$

In the above equation,

-The term rand ( )*(pbesti $-\mathrm{Pi}(\mathrm{u}))$ is called particle memory influence

-The term rand ( )*(gbesti -Pi (u)) is called swarm influence

$-\mathrm{Vi}(\mathrm{u})$ which is the velocity of ith particle at iteration ' $u$ ' must lie in the range,

\section{$V i \min \leq V i \leq V i \max$}

- The parameter Vmax determines the resolution, or fitness, with which regions are to be searched between the present position and the target position

- .If Vmax is too high; particles may fly past good solutions. If Vmin is too small, particles may not explore sufficiently beyond local solutions.

- In many experiences with PSO, Vmax was often set at 10-20\% of the dynamic range on each dimension.

- The constants $\mathrm{C} 1$ and $\mathrm{C} 2$ pull each particle towards pbest and gbest positions.

- Low values allow particles to roam far from the target regions before being tugged back. On the other hand, high values result in abrupt movement towards, or past, target regions.

- The acceleration constants $\mathrm{C} 1$ and $\mathrm{C} 2$ are often set to be 2.0 according to past experiences

- Suitable selection of inertia weight ' $\omega$ ' provides a balance between global and local explorations, thus requiring less iteration on average to find a sufficiently optimal solution.

- In general, the inertia weight $w$ is set according to the following equation,

$W=W \max -[W \max -W \min /$ ITER $\max ] *$ ITER.

Where $\mathrm{W}$-is the inertia weighting factor

Wmax - maximum value of weighting factor

Wmin - minimum value of weighting factor

ITERmax - maximum number of iterations

ITER - current number of iteration

The PSO algorithm can be best described in general as follows: 
1) For each particle, the position and velocity vectors will be randomly initialized with the same size as the problem dimension.

2) Measure the fitness of each particle (pbest) and store the particle with the best fitness (gbest) value.

3) Update velocity and position vectors for each particle.

4) Repeat steps until a termination criterion is satisfied. In addition to traditional gradient-based optimization algorithms, there are many other heuristic techniques that compete with PSO such as genetic algorithm, simulated annealing, evolutionary programming, and most recently ant colony optimization

The advantages of aforementioned algorithms over PSO are the following

- The availability of commercial versions of some algorithms like Matlab (genetic algorithm) and Excel premium solver (evolutionary programming).

- The extensive collection of books and research literatures, especially in the case of genetic algorithm and evolutionary programming, which cover these competing methods. Despite the simplicity of the PSO concept and implementation, its superiority is proven when compared with other techniques in many different application areas.

However, unlike GA, PSO has no evolution operators such as crossover and mutation. In PSO, the potential solutions, called particles, fly through the problem space by following the current optimum particles. The detailed information will be given in following sections. Compared to GA, the advantages of PSO are that PSO is easy to implement and there are few parameters to adjust. PSO has been successfully applied in many areas: function optimization, artificial neural network training, fuzzy system control, and other areas where GA can be applied.

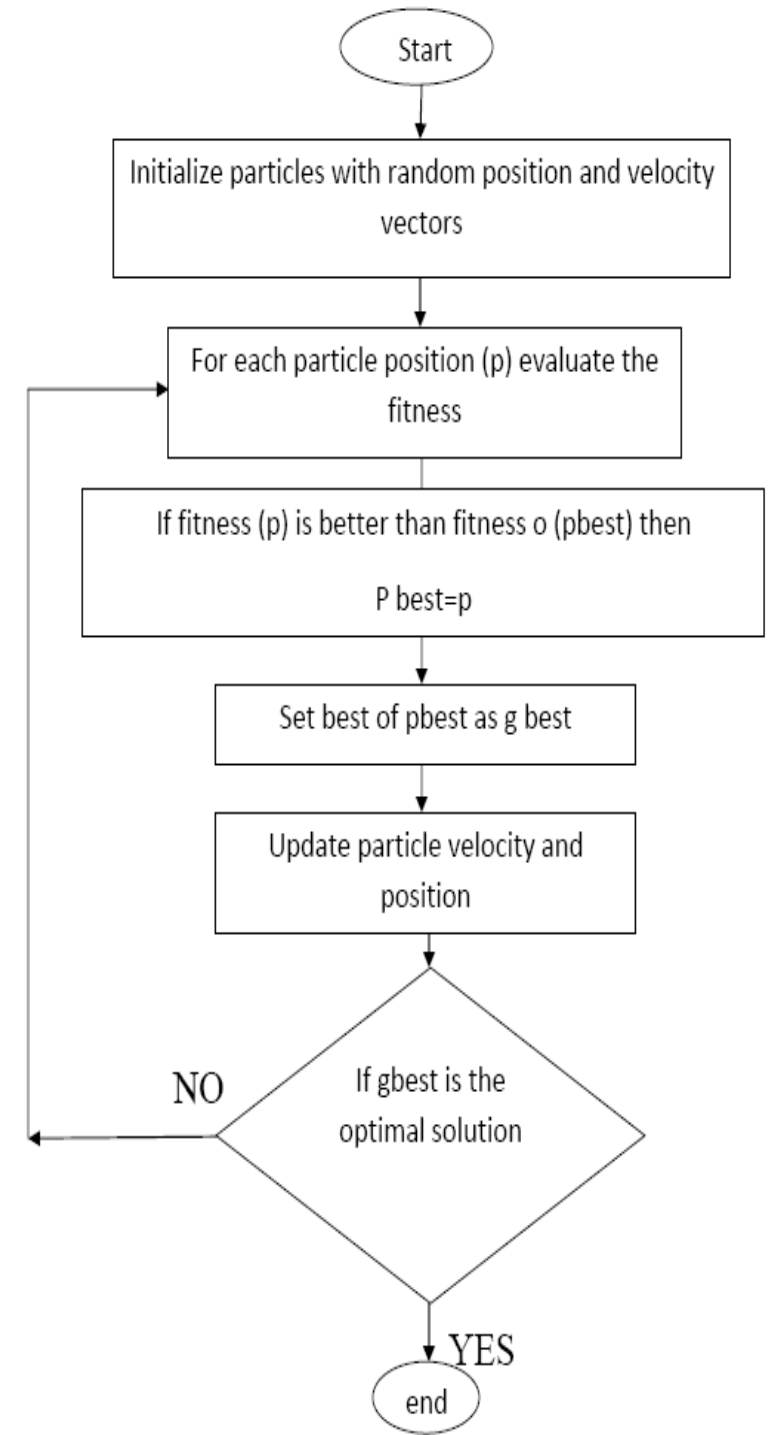

Figure 1: Flow chart

\subsection{Multi Agent System}

A multi-agent system (MAS) is a system composed of multiple interacting intelligent_agents within an environment. Multi-agent systems can be used to solve problems that are difficult or impossible for an individual agent or a monolithic_system to solve [7]. Intelligence may include some methodic, functional, procedural or algorithmic search, find and processing approach. Topics where multi-agent systems research may deliver an appropriate approach include online trading, disaster response, and modeling social structures. Agents have a certain level of autonomy, which means that they can take decisions without a central controller or commander. To achieve this, they are driven by a set of tendencies.

Multi-agent systems consist of agents and their environment. Typically multi-agent systems research refers to software agents. However, the agents in a multi-agent system could equally well be robots, humans or human teams. A multiagent system may contain combined human-agent teams.

Agents can be divided into different types

- Very simple like: passive agents or agent without goals 
- Active agents with simple goals

- $\quad$ Or very complex agents

Environment also can be divided into

- Virtual Environment

- Discrete Environment

- Continuous Environment

Agent environments can be organized according to various properties like: accessibility (depending on if it is possible to gather complete information about the environment), determinism (if an action performed in the environment causes a definite effect), dynamics (how many entities influence the environment in the moment), discreteness (whether the number of possible actions in the environment is finite), periodicity (whether agent actions in certain time periods influence other periods), and dimensionality (whether spatial characteristics are important factors of the environment and the agent considers space in its decision making) [7]

Multi Agent System technology provides an opportunity to compute and optimize many complicated problems. Agents in Multi agent system act collection of a society and they compete to achieve their own individual goals as well as the common goal. This feature matches the essential nature of a particle in Particle Swarm Optimization. Hence, this paper combines PSO and MAS to form a new optimal algorithm [8].

The main elements included in Multi Agent System is listed below

a) The meaning and purpose of each agent

b) An environment where all agents live

c) The definition of a local environment

d) A set of behavioral rules

\subsubsection{Multi Agent based Particle Swarm Optimization}

In Multi Agent System and PSO, integrates multi agent system and PSO to form a multi agent-based PSO approach (MAPSO), for solving the optimization problem. In MAPSO, an agent represents a particle to PSO and a candidate solution to the optimization problem. All agents live in a lattice-like environment, with each agent fixed on a lattice point

In order to obtain optimal solution quickly, compete and cooperate with their neighbors, and also use their knowledge. These agent-agent interactions and evolution mechanism of PSO in a lattice-like environment, this method can find the high quality solutions with the faster convergence characteristics in a good computation time [14].

The Multi Agent based Particle Swarm Optimization method for solving load shedding problems. In MAPSO, an agent represents not only a candidate solution to the optimization problem but also a particle to PSO. Firstly, a lattice-like environment is constructed, with each agent fixed on a latticepoint.

In MAPSO, an agent represents a candidate solution to the optimization problem and is a particle to PSO. Hence, agent has a fitness value to the optimization problem. For solving Load Shedding problem, its fitness value is the value of the active power loss in the transmission network $f(\alpha)=f q=\sum_{k \in N r}$ Pkloss $=\sum_{k \in N r} g k\left(V i^{\wedge} 2+V j^{\wedge} 2-2 V i V j \cos \theta i j\right) \ldots \ldots . .12$

The purpose of is to minimize the real power transmission losses and keep all the voltages within the limits as much as possible. In Load Shedding problem, each agent carries all control variables to be optimized.

In Multi Agent System, all agents live in an environment and environment is organized as a lattice-like structure. In the environment, each agent is fixed on a lattice-point and each circle represents an agent [15], the data in circle represents its position in the environment. Due to representation of a particle in PSO, each agent in its database contains two data, particle's current velocity and its coordinates in the search space.

Each agent can only sense its local environment in Multi Agent System. The neighbors of $\alpha_{\mathrm{ij}}$ and $\mathrm{N}_{\mathrm{ij}}$ is defined as [8]

$\mathrm{N} i, j=\{\alpha i 1, j, \alpha i, j 1, \alpha i 2, j, \alpha i, j 2\}$

Each agent has some behaviors. In MAPSO, each agent firstly competes and cooperates with its neighbors to diffuse its useful information to the whole environment, and it can also use evolution mechanism of PSO and its knowledge

\subsubsection{MAPSO Based Load Shedding Method}

In previous sections, some load could be shed in emergency condition to protect the other load. The main problem is there is no idea about where the Load could be shedding and how much could be shedding. So Load Shedding bring up large economic lost. Hence this algorithm helps to solve these types of problems and load shedding strategy can be optimized with the PSO

The mathematical model is

$\min f(P, Q)=\sum_{i \varepsilon 1} P g i-\sum_{j \varepsilon L} P l j+\sum_{j \varepsilon L} W l j\left(P l j-P l j^{\prime}\right) \ldots \ldots \ldots 14$

$P i=V i \sum_{i=1}^{n} V j(G i j \cos \theta i j+B i j \sin \theta i)$

From these equations, the reactive load always variable with the active load. The reactive load will decrease with the active load with the Power factor unchanged. While the equality constraints could be eliminated. The generator outputs and the loads are control variables, and they could be bounded after updating the position of particles [8].

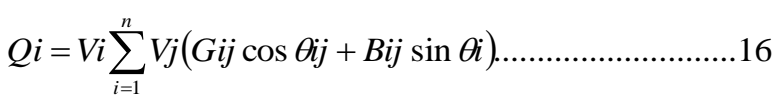

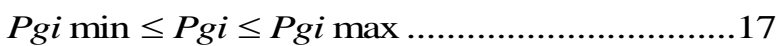

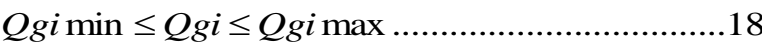

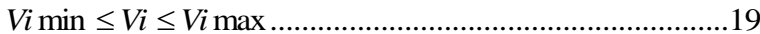

$-F i \min \leq F i \leq$ Fii $\max \ldots \ldots \ldots \ldots \ldots \ldots \ldots \ldots \ldots \ldots \ldots \ldots \ldots . . .20$

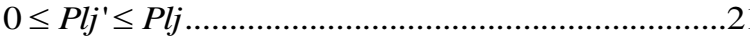

The control variable $\mathrm{P}$ and $\mathrm{Q}$ not only contains the output of generator but also the active load. $\mathrm{P}_{\mathrm{lj}}$ is demand power at bus $\mathrm{j}$, but only $\mathrm{P}_{\mathrm{lj}}$ ' is supplied, so the amount of load $\mathrm{P}_{\mathrm{lj}} \mathrm{P}_{\mathrm{lj}}$, is shed, the factor $\mathrm{w}_{\mathrm{lj}}$ is the economical lost coefficient for the load $\mathrm{j}$. It 
must be pointed that the reactive load always variable with the active load. In this paper, the reactive load will decrease with active load with the Power factor remaining unchanged.

Let

$$
\begin{aligned}
& X i=\left(X i^{\wedge} 1, X i^{\wedge} 2, \ldots \ldots . . . X i^{\wedge} n\right) \ldots \ldots \ldots \ldots \ldots \ldots \ldots \ldots \ldots \ldots . . .22 \\
& X i, t+1=X i, t+V i, t+1
\end{aligned}
$$

Suppose $\mathrm{x}$ is the control variable vector consisting of $\mathrm{P}$ and $\mathrm{Q}$, etc., and it's dimension is $\mathrm{x}^{\mathrm{n}}$. For particle $\mathrm{i}$,

Then it becomes

$$
\begin{aligned}
& X^{\wedge} j \min \ldots \ldots . . i f X i^{\wedge} j<X \min ^{\wedge} j \\
X i^{\wedge} 1= & X \max ^{\wedge} j \ldots . . . i f X i^{\wedge} j>X \max ^{\wedge} j \ldots \\
& X i^{\wedge} j \ldots \ldots \ldots \ldots . . . o t h e r s
\end{aligned}
$$

The power equation becomes

$$
\begin{aligned}
\min f(x)= & \sum_{i \& G} P g i-\sum_{j \varepsilon L} P l j \\
& +W h \sum_{J=1} \max (0, h j(x)) . \\
& +\sum W l j\left(P l j-P l j^{\prime}\right)
\end{aligned}
$$

From these equations the aim function consists of three parts. The first part is the Power loss, the second part is the penalty of violation of constraints and the third part is the economic cost of the Load Shedding. The inequality constraints contains voltage constraint and transmission capacity constraints

\subsubsection{Implementation of Multi Agent based PSO for Load Shedding Problems}

In MAPSO are utilized to simulate the behavior of agents and realize the purposes. It reduces the computational cost, the operator is performed only the agent with the minimum fitness value in each generation. But it has an important effect on the performance of MAPSO. The proposed method can quickly and accurately converge to the optimal solutions for Load shedding optimization approach.

Mainly considered there are seven steps

Step I: Consider input parameters of system and algorithm and also specify lower and upper boundaries of each variable

Step II: Generate the lattice like environment and initialize randomly each agent.

Step III: Calculate the fitness of each agent with respect to the Load Shedding analysis

Step IV: Then update the time counter

Step V: Perform the neighborhood competition and cooperation operator on each agent.

Step VI: After that execute the PSO operator and adjust its position in the search space on each agent according to the velocity equations.

Step VII: Find the best agent with the minimum fitness value, and then perform the self-learning operator.

\section{SIMULATION STUDIES}

In this paper Multi Agent Based PSO is used for controlling the Load shedding problems. The Load Shedding is assigned with the priority of customers and make the generation is $1000 \mathrm{MW}$. Consider each and every agent they have different priorities

First assign the lower priority, obtained the corresponding optimized Load value for the proper Load Shedding and referred the following equations. Where A, B, C are constants and $\mathrm{P}$ is the power got from the previous equations. Initial value of total cost is taken as 0 .

$$
\begin{aligned}
& \text { Fuel } \cos t=A+B * P i+C^{*} P j * P j \ldots \ldots \ldots \ldots \ldots \ldots \ldots \ldots . . .26 \\
& \text { Total } \cos t=\text { total } \cos t(\text { initial })+\text { Fuel } \cos t \ldots \ldots \ldots \ldots . .27
\end{aligned}
$$

The consumers are represented as $\mathrm{C} 1, \mathrm{C} 2, \mathrm{C} 3, \mathrm{C} 4$, and $\mathrm{C} 5$, each consumer have different priority. It may be lower, medium or high, consider these priorities and got the optimum value corresponding to their Load Demand

Total cost of Each Generation unit obtained from the simulation, $\mathrm{C} 1$ corresponds to the total cost is $2.6333 \mathrm{e}+003$; this cost value depends upon the consumers priority. Consider there are 5 parameters or consumers.

Similarly total cost of consumer C2 is $4.3752 \mathrm{e}+003$

Total cost of consumer C3 is $1.3734 \mathrm{e}+004$

Total cost of consumer $\mathrm{C} 4$ is $2.2673 \mathrm{e}+004$

Total cost of consumer $\mathrm{C} 5$ is $3.7808 \mathrm{e}+004$

The Load Schedule corresponding to the generation

Load $=69.7300$

Load $=95.1410 \quad 50.8221$

Load $=263.4590 \quad 219.1400 \quad 336.6359$

Load $=414.0326 \quad 369.7136 \quad 487.2095 \quad 301.1472$

Load $=749.4485 \quad 705.1295 \quad 822.6254 \quad 636.5631670 .8318$

The Load Shedding is assigned with the priority of customers and make the generation is 1000MW. Consider each and every agent they have different priorities First assign the lower priority, obtained the corresponding optimized Load value for the Load shedding [4]. The load values vary randomly. The Load Schedule obtained from the following equations,

Loadshed $=((d v / d q) /$ sum $) *$ Pdiff .28

Where,

Pdiff $=($ dfdtfinal $* 2 * H / 60) * 1000$

$d q, d v=V i * Y i j * \sin ((\Delta P d i)-(\Delta Q d i))-(\delta i-\delta j))$

$d v / d q=1 /(d q / d v)$

Obtained the total cost of each generation unit of the entire system and Corresponding Load Schedule is obtained. There are two cases, one is the system with losses and another one is system without losses. In both cases should get the total 
cost and Load Schedule. In this paper result represents the values without losses, so the error value is zero.

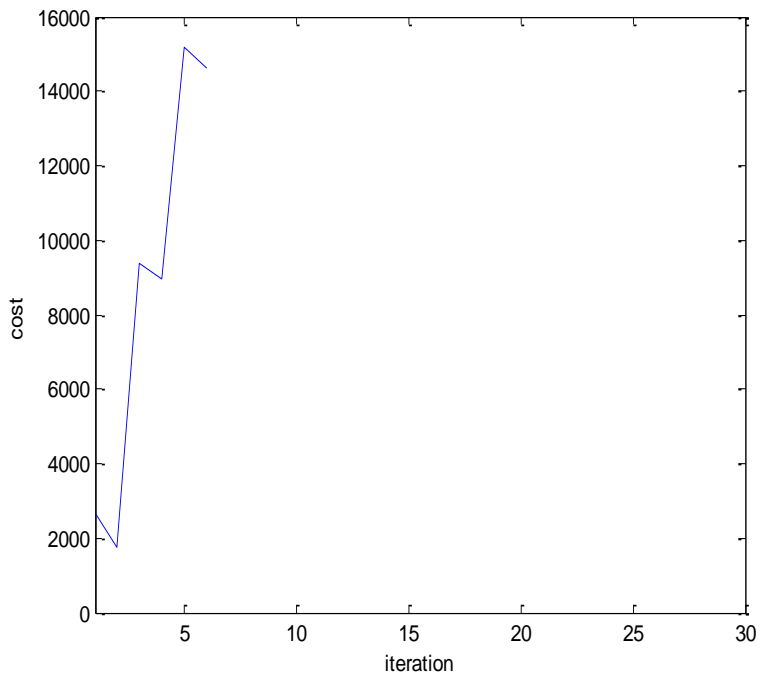

Figure 2: Cost Vs Iteration (30) without loss

The consumers are represented as $\mathrm{C} 1, \mathrm{C} 2, \mathrm{C} 3, \mathrm{C} 4$, and C5, each consumer have different priority. It may be lower, medium or high, consider their priorities and got the optimum value of their corresponding Load Schedule.

This graph is plotted between scheduling cost and its irrespective iteration. In this method 3 generating units are committed along with the system. This plot shows the optimum value of the fuel cost value. Number of iteration is 30 . If the number of iteration is 30 , the graph is shown below.

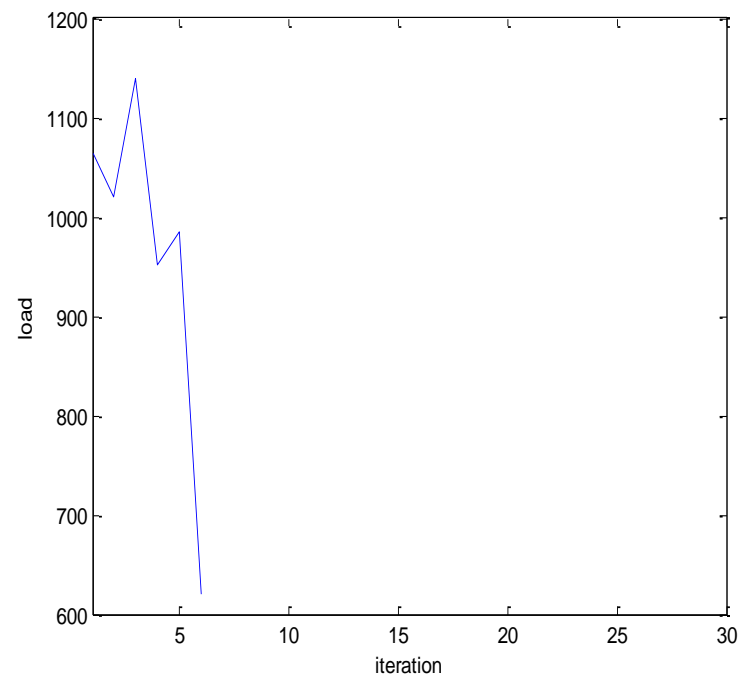

Figure 3: Load Vs Iteration (without loss)

This graph shows the graph between load and respective iteration. The Load Shedding is assigned with the priority. Make the generation is $1000 \mathrm{MW}$. There are different priorities assigned with each agent. First consider the lower priority, got the corresponding optimized Load value for the Load shedding [3]. The graph between net power and iteration is shown below. Sometimes the load value is taken as corresponding net power of each agent.

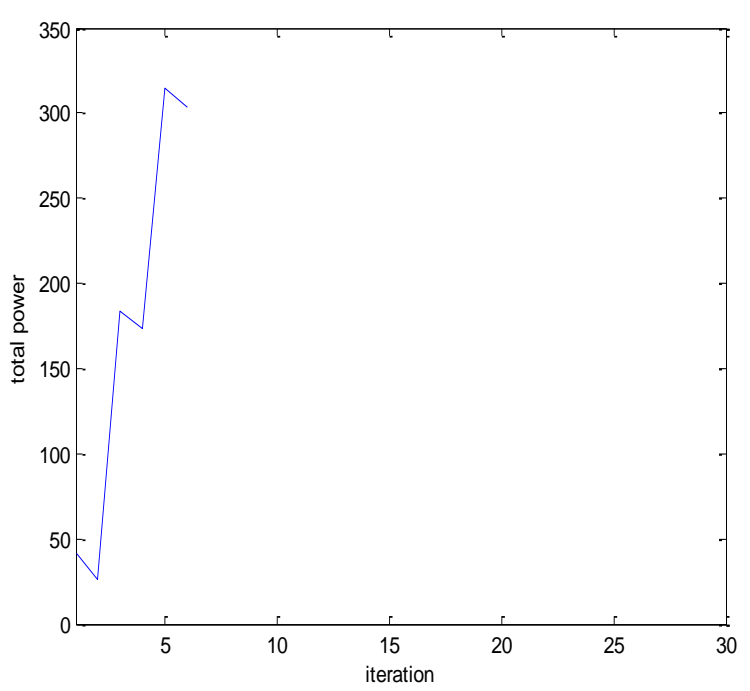

Figure 4: Total net Power Vs Iteration

\section{CONCLUSION}

In this paper, a stable MAS-based Particle Swarm Optimization (MAPSO) is proposed for controlling the Load Shedding Problems. Even though each agent only communicates with its direct neighbors, global information that is necessary for distributed load shedding can be discovered. Load Shedding is assigned with the priority of each customer. Compared with existing MAS-based researches in power systems and obtained the total cost of each generation unit of the entire system. Corresponding Load Schedule is obtained. There are two cases, one is the system with losses and another one is system without losses. In both cases obtained the total cost and Load schedule. The MAPSO method is ability to finding the high quality solutions with fast convergence properties.

For the future extension of the project work, a Genetic Algorithm will be introduces for controlling the Load Shedding problems. This algorithm will produce global information with respect to the proper Load Shedding decisions. The Genetic Algorithm handles a population of possible solution. It starts with randomly generated population. It will be apply to the 30 bus system, and will compare both 5 bus and 30 bus system in future.

\section{REFERENCES}

[1] F. Shokooh, J. J. Dai, S. Shokooh, J. Taster, H. Castro, T. Khandelwal, and G. Donner, "An intelligent load shedding (ILS) system application in a large industrial facility," in Proc. 14th IAS Annu. Meeting, 2005, vol. 1, pp. 417-425.

[2] S. Borg, G. Andersson, D. J. Hill, and I. A. Hiskens, "On influence of load modeling for under voltage load shedding studies," IEEE Trans. Power Syst., vol. 13, no. 2, pp. 395400, May 1998

[3] B. Otomega,M. Glavic, and T. Van Cutsem, "Distributed under voltage load shedding," IEEE Trans. Power Syst., vol. 22, no. 4, pp. 2283-2284, Nov. 2007.

[4] V. V. Terzija, "Adaptive under frequency load shedding based on the magnitude of the disturbance estimation," IEEE Trans. Power Syst., vol. 21, no. 3, pp. 1260-1266, Aug. 2006 
[5] B. Hamid, O. Abderrahmane, G. Nadir, M. Farid, and E. M. Nikos, "A new approach applied to adaptive centralized load shedding scheme," in Proc. 8th WSEAS Int. Conf., 2009, pp. 28-33.

[6] H. Seyedi and M. Sanaye-Pasand, "New centralized adaptive load-shedding algorithms to mitigate power system blackouts," IET Gen., Transm., Distrib., vol. 3, no. 1, pp. 99-114, 2009.

[7] M. Wooldridge, "An Introduction to Multiagent Systems". New York: Wiley, 2002.

[8] Zhao B, Guo C. X, and Y. J. Cao, "A Multiagent-Based Particle Swarm Optimization Approach for Optimal Reactive Power Dispatch" IEEE Transactions on Power Systems, vol. 20, no. 2, may 2005.

[9] Yinliang Xu, Wenxin Liu, "Stable Multi-Agent-Based Load Shedding Algorithm for Power Systems" IEEE Transactions on Power Systems, vol. 26, no. 4, November 2011

[10] T. L. Baldwin and S. A. Lewis, "Distribution load flow methods for shipboard power systems," IEEE Trans. Ind. Appl., vol. 40, no. 5, pp. 1183-1190, Sep./Oct. 2004.
[11] Yamille del Valle, Ganesh Kumar Venayagamoorthy, Salman Mohagheghi, Jean-Carlos Hernandez, and Ronald G. Harley, "Particle Swarm Optimization: Basic Concepts, Variants and Applications in Power Systems" IEEE Transactions on Evolutionary computation, vol. 12, no. 2, april 200

[12] J. M. Solanki, S. Khushalani, and N. N. Schulz, "A multiagent solution to distribution systems restoration," IEEE Trans. Power Syst., vol. 22, no. 3, pp. 1026-1034, Aug. 2007.

[13] K. Huang, D. A. Cartes, and S. K. Srivastava, "A multiagent-based algorithm for ring-structured shipboard power system reconfiguration," IEEE Trans. Syst., Man, Cybern. C, Appl. Rev., vol. 37, no. 5, pp. 1016-1021, Sep. 2007.

[14] F. Xiao, L. Wang, and Y. Jia, "Fast information sharing in networks of autonomous agent," in Proc. American Control Conf., 2008, pp. 4388-4393.

[15] R. Olfati-Saber and R. M. Murray, "Consensus problems in networks of agents with switching topology and timedelays," IEEE Trans. Autom. Control, vol. 49, no. 9, pp 1520-1533, Sep. 2004. 